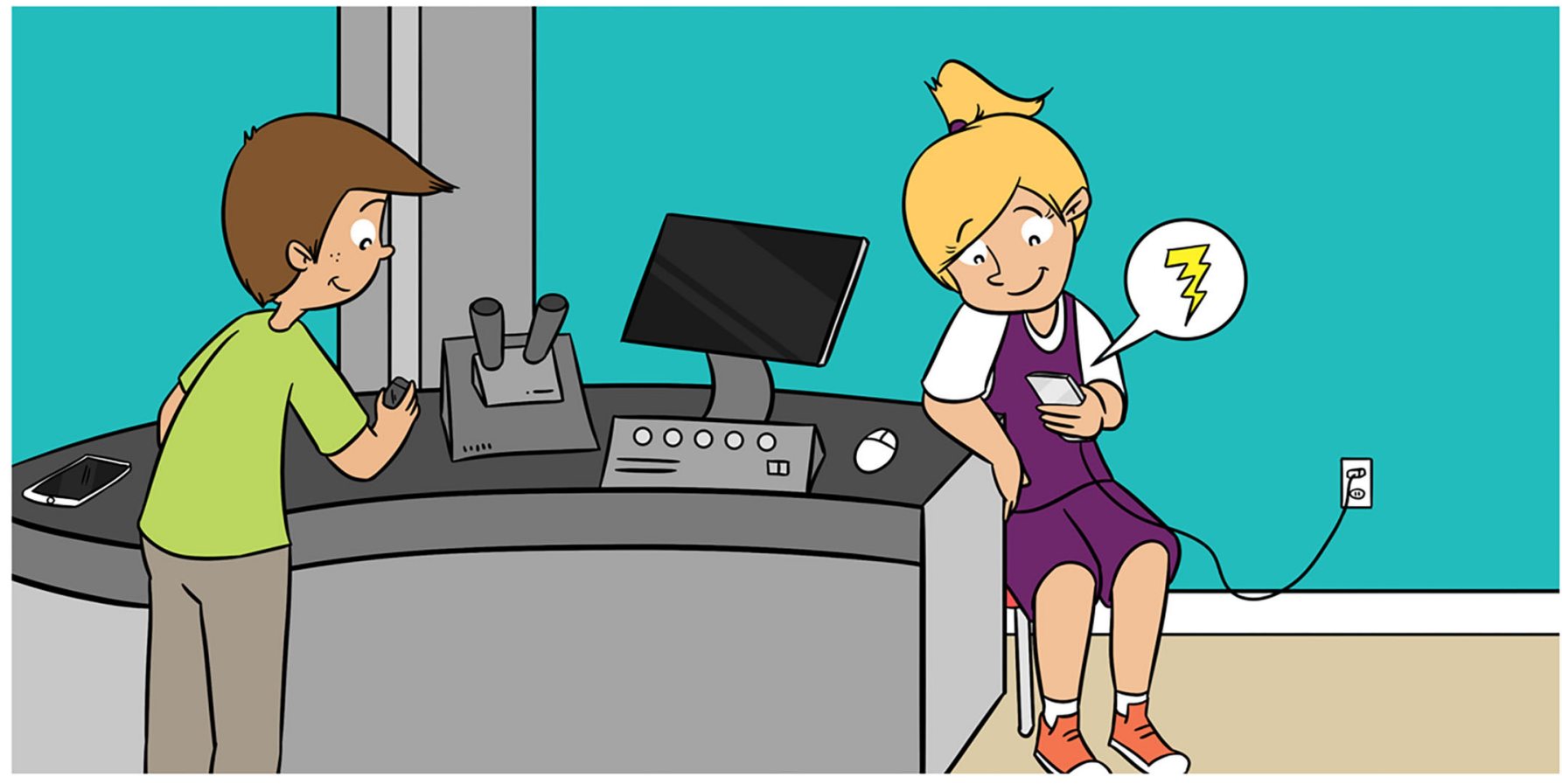

\title{
TAMING LITHIUM: MAKING FUTURE BATTERIES SAFER
}

\section{David Rehnlund $^{1,2^{*}}$ and Julia Maibach ${ }^{3}$ \\ ${ }^{1}$ Department of Chemistry - Angström Laboratory, Uppsala University, Uppsala, Sweden \\ ${ }^{2}$ Department of Applied Biology, Institute for Applied Biosciences, Karlsruhe Institute of Technology, Karlsruhe, Germany ${ }^{3}$ Institute for Applied Materials, Karlsruhe Institute of Technology, Karlsruhe, Germany}

YOUNG REVIEWER:

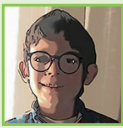

UMBERTO

AGE: 10
Can you imagine having your smartphone, TV remote control, or drone connected to an electric cable all the time? This was the reality before 1991, when rechargeable lithium batteries were invented and changed our world. These small devices can power all electronics, making them mobile and wireless. However, our electronic devices are getting more advanced and require more electricity. Battery scientists are working hard to develop new and improved batteries that can power your smartphone for even longer before you need to charge it again. The dream is to use lithium metal, which can store a lot of electricity. Sadly, lithium is very difficult to control and can grow into wires that make the battery unsafe to use. In our recent study, we investigated how lithium grows inside batteries and discovered that it is possible to tame lithium and make it safe for use in future batteries. 


\section{ELECTRODES}

Solid material that stores ions by binding them to electrons. In the electrode, the current is conducted by moving electrons.

\section{ANODE}

One of the electrodes in a battery, the negatively charged pole. Here, ions and electrons are released during discharge.

\section{CATHODE}

One of the electrodes in a battery, the positively charged pole. Here, ions and electrons are absorbed and stored during discharge.

\section{ELECTROLYTE}

Liquid that contains a salt dissolved in a solvent, for example table salt in water. The electrolyte can conduct a current by moving ions.

ION

Atom or group of atoms with an electric charge that is either positive or negative. Lithium ions have a positive charge.

\section{ELECTRON}

Electrons are even smaller than atoms and have a negative electric charge.

CURRENT

When electrons move in one direction, this electron flow is called a current. When you divide the current by the electrode area it is called current density. The current is the driving force of all electronic devices, such as your smartphone.

\section{HOW DOES A BATTERY WORK?}

Batteries are small electronic devices that power everything from smartphones to electric cars. Batteries release electricity by converting stored chemical energy into electrical energy. Inside the battery there are two electrodes, called the anode and cathode, which are separated by a liquid called the electrolyte. There are two important particles that drive a battery, ions, and electrons. The ions, which have a positive charge, can swim through the electrolyte liquid, but the electrons, which have a negative charge, cannot swim and must run through cables when moving between the two electrodes.

As we illustrate in Figure 1, there are two ways to "operate" a battery: charging (storing energy) and discharging (using the stored energy). When you charge the battery in your phone, you provide energy to the battery that pumps the electrons into the anode. There, the electrons can connect to their ion partners. When you use the battery in your phone, the electrons want to rush to the cathode. We call this flow of electrons a current. The current is guided by electronic circuits to power devices like your phone. Eventually, the current runs out and the battery is discharged. Certain batteries can be recharged by forcing the electrons back into the anode. The lithium battery is the best and most used rechargeable battery.

\section{BUILDING BATTERIES FOR THE FUTURE}

As our electronic devices become more advanced, their appetite for power increases. Battery researchers around the world are working hard to create new and improved batteries. There are many parts of a battery that can be improved, but the most important goal is to increase the amount of energy that can be stored in it.

To increase the energy storage in batteries, we need to find better electrodes. One very promising electrode uses the element lithium. Lithium is the lightest metal on earth. It is also very reactive, which means it is the metal that most willingly releases electrons. This makes lithium perfect for portable batteries. Lithium was first used in a battery invented by Professor Whittingham in the 1970s [1]. Sadly, lithium is very difficult to control and can cause the battery to burn. Professors Yoshino [2] and Goodenough [3] changed the electrodes in the first lithium battery to produce the safe version we now use in our daily lives. These important achievements awarded the three professors the Nobel Prize in Chemistry in 2019.

\section{THE LITHIUM PROBLEM}

Scientists across the world have never let go of the dream of using lithium to make better batteries. For nearly 40 years, battery scientists 
Figure 1

A lithium ion battery from a smartphone (left) and the inside of a battery (right). When charging, negative electrons are moved by a power source from the cathode to the anode. When discharging to power a phone, negative electrons flow naturally from the anode to the cathode. In both cases, the positive ions follow the same direction as the negative electrons but swim through the electrolyte.

\section{ATOM}

A very small particle without any electric charge. Atoms are the basic building blocks of all matter in the universe.

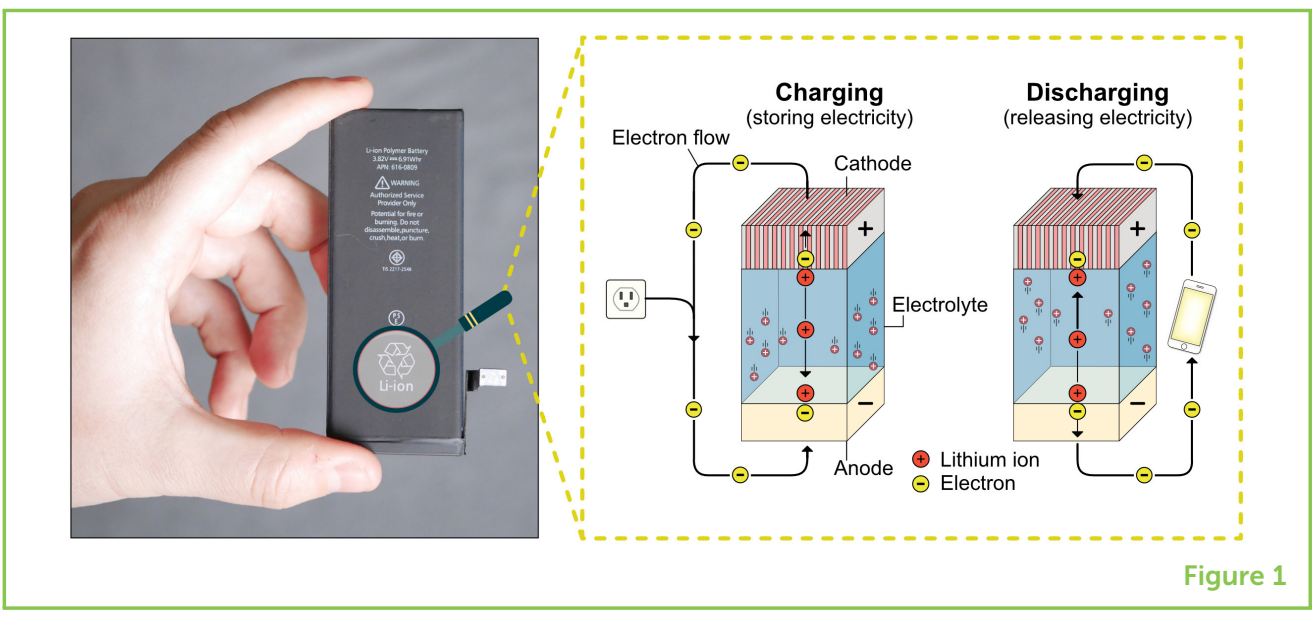

have tried all possible ways to make lithium safer. The problem is very difficult, and we have not yet found a solution. So why is lithium unsafe? It likes to grow into wires inside the battery!

We recently investigated this lithium problem to find a way of controlling how lithium grows [4]. We started by investigating how lithium wires are formed on the anode. Figure 2 shows the growth of lithium wires. When a positive lithium ion meets a negative electron, they react and form a neutral (no electrical charge) lithium atom on the electrode surface. More and more ions meet their electron partners and form atoms on the surface. Normally, this process happens very quickly and the newly formed lithium atoms have very little time to arrange themselves in even layers. Instead, the atoms build upon each other and grow as wires into the electrolyte. The lithium ions now have a shorter path to swim to the growing lithium wire. The wires will attract nearby ions and grow faster than the rest of the electrode surface. Eventually, the lithium wires grow so long that they connect to the opposite electrode side. Electrons can now move down the wire instead of taking the detour around the battery (through the electronic circuits of the device) to reach the lithium electrode. This shorter path is called a short-circuit and it will make the battery unsafe.

How can this dangerous problem be prevented? We studied this by looking at lithium electrodes inside a battery [4]. The battery was first charged to grow new lithium atoms on the anode surface. Then we opened the battery and removed the lithium electrode. We looked at the electrode surface with a special electron microscope that can see objects as small as a few atoms. Next, we varied the ingredients in the battery and the way electricity was fed into the battery during charging. We found three main factors that control how lithium grows (Figure 3). 
Figure 2

When lithium ions and electrons meet and react in an uncontrolled way, lithium atoms are formed and they pile up on each other, forming lithium wires. When a lithium wire connects the two electrodes inside a battery, this creates a shorter path for the electrons. The electrons rush over from cathode to the anode through the wire, in a so-called short-circuit. This is dangerous because the wires become hot and can cause the electrolyte to catch fire.

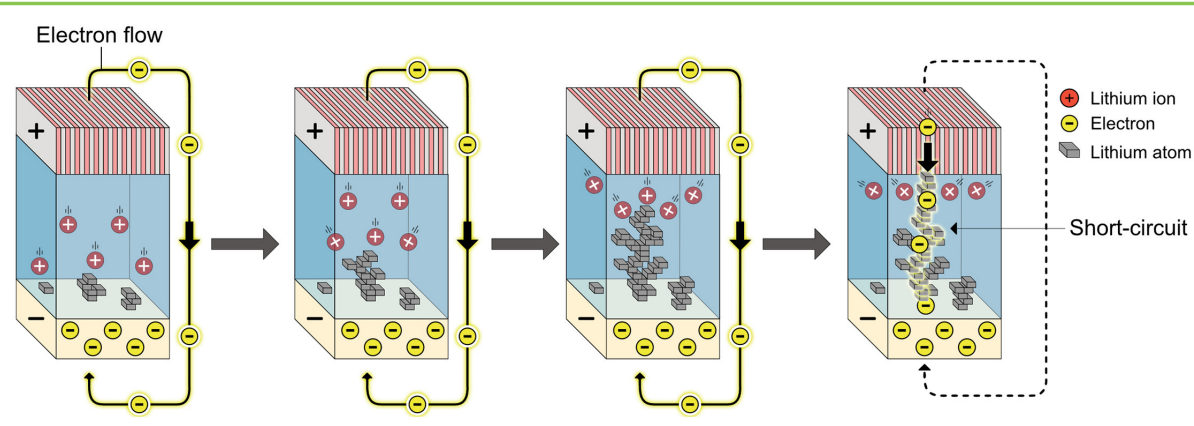

Figure 2

\section{DECREASING THE FLOW OF ELECTRONS}

The first factor that affects the growth of lithium wires is current density, which is the flow of electrons through the battery. High current density means many electrons moving between the electrodes at the same time. When few electrons move, then the current is low. The current is like traffic in a city. Without any speed limits, the electrons will rush to the electrode and grab any partner ion that comes close to the electrode surface. With this high current, the electron-ion pairs will land wherever they can. If we instead add speed limits in the battery, the current density will be low. This means fewer electrons on the electrode surface. The electrons now have time to move around and find a comfy spot where they can grab an ion and form a lithium atom. Low current density gives more time for the lithium atoms to be placed in an ordered pattern. This means we can help lithium to grow into layers instead of wires by adding speed limits for the electrons. Layered lithium makes the battery safe to use, because no wires can grow between anode and cathode to cause a dangerous short-circuit.

\section{DECREASING LITHIUM IONS IN THE ELECTROLYTE}

The second factor affecting the growth of lithium wires is the ion concentration in the electrolyte. High concentration means more ions in the electrolyte. When the electrolyte is full of ions, they will swim around looking for electrons to react with. Like we saw with the electrons, this behavior causes atoms to quickly grow on top of each other, into wires. If we instead lower the ion concentration, then fewer ions will swim around, which gives them time to find a good spot on the electrode surface. It is important to understand that, when lowering the ion concentration, we do not change the stored energy in the battery. The energy in the battery depends on how much lithium we have in the electrodes, not in the electrolyte, which means that we can change the ion concentration to control how many ions move at the same time, without making the battery worse. 
Figure 3

Current density, ion concentration, and nuclei density all control how lithium grows in a battery. When current density and ion concentration are high and the number of nuclei "parking spaces" is low, wires form. However, when there are many parking spaces and current density and ion concentration are low, safer, flat layers form. At the bottom, you can see electron microscope photographs of the lithium surface from battery 1 and battery 2 . which show the differences in lithium wire formation under these two sets of conditions.

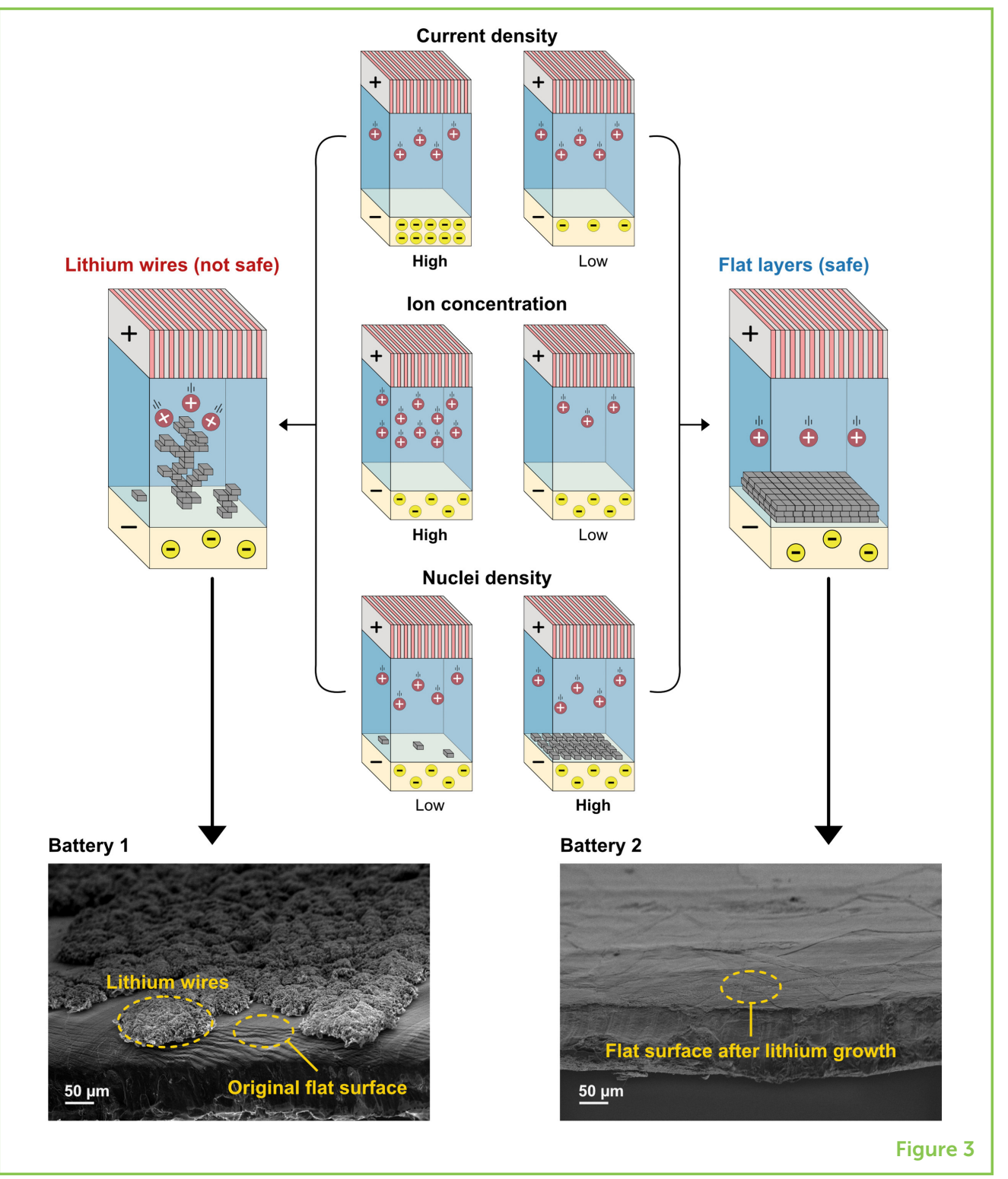

\section{INCREASING THE NUMBER OF "PARKING SPOTS"}

The third factor affecting the growth of lithium wires is the nuclei density on the electrode surface. Nuclei are small islands of atoms that act as spots for lithium growth to start. Nuclei are like parking spots for atoms. Low nuclei density means few parking spots, which forces the lithium atoms to pile on top of each other. If we increase the nuclei density and offer more parking spots, then the lithium atoms can all find a spot and neatly arrange themselves. High nuclei density can help the atoms to grow into layers, not wires.

\section{OUR TEST BATTERY}

In our research, we focused on changing the ion concentration and nuclei density to see if we could decrease the formation of 
lithium wires. We built two similar batteries. Battery 1 had a high ion concentration and low nuclei density. Battery 2 had a low ion concentration and high nuclei density. The two batteries were then charged and discharged. Afterwards, we took the batteries apart and looked at the lithium anode surface. In Figure 3, we show the photographs taken with the electron microscope so you can see what the surfaces of these batteries looked like. The electrode from battery 1 was covered in a web of lithium wires, which are about 1,000 times smaller than a strand of hair. The electrode from battery 2 had a very smooth surface. This means that lithium had grown into layers.

\section{OUR RECIPE FOR A SAFE LITHIUM BATTERY}

To tame lithium and make safe batteries, we must slow down the growth of lithium wires. This can be done by reducing the number of electrons and ions moving in the battery at the same time. Lithium will also grow in more controlled into layers if we add many nuclei on the electrode surface. With our new strategy, the electrode surface is very smooth and shows very few wires. Without our strategy, the lithium surface was completely covered by wires. This proves that it is possible to control lithium electrodes, which is a very exciting new discovery. We hope that the new knowledge will help battery researchers develop new and improved batteries. It is our goal that this next generation of batteries will store more energy so that they can power your phone or car even longer before needing to be charged again.

\section{ORIGINAL SOURCE ARTICLE}

Rehnlund, D., Ihrfors, C., Maibach, J., and Nyholm, L. 2018. Dendrite-free lithium electrode cycling via controlled nucleation in low $\mathrm{LiPF}_{6}$ concentration electrolytes. Mater. Today 21:1010-8. doi: 10.1016/j.mattod.2018.08.003

\section{REFERENCES}

1. Whittingham, M. S. 1976. Electrical energy storage and intercalation chemistry. Science 192:1126-7. doi: 10.1126/science.192.4244.1126

2. Yoshino, A. 2012. The birth of the lithium-ion battery. Angew. Chem. Int. Ed. 51:5798-800. doi: 10.1002/anie.201105006

3. Mizushima, K., Jones, P. C., Wiseman, P. J., and Goodenough, J. B. 1980. $\mathrm{Li}_{x} \mathrm{CoO}_{2}(0<x<-1)$ : a new cathode material for batteries of high energy density. Mater. Res. Bull. 15:783-9. doi: 10.1016/0025-5408(80)90012-4

4. Rehnlund, D., Ihrfors, C., Maibach, J., and Nyholm, L. 2018. Dendrite-free lithium electrode cycling via controlled nucleation in low LiPF 6 concentration electrolytes. Mater. Today 21:1010-8. doi: 10.1016/j.mattod.2018.08.003 
SUBMITTED: 09 December 2019; ACCEPTED: 03 August 2020;

PUBLISHED ONLINE: 04 September 2020.

EDITED BY: Noemie Ott, Swiss Federal Laboratories for Materials Science and Technology, Switzerland

CITATION: Rehnlund D and Maibach J (2020) Taming Lithium: Making Future Batteries Safer. Front. Young Minds 8:118. doi: 10.3389/frym.2020.00118

CONFLICT OF INTEREST: DR is an inventor on a patent application entitled "An electrochemical device and method for charging the electro-chemical device."

The remaining author declares that the research was conducted in the absence of any commercial or financial relationships that could be construed as a potential conflict of interest.

COPYRIGHT (c) 2020 Rehnlund and Maibach. This is an open-access article distributed under the terms of the Creative Commons Attribution License (CC BY). The use, distribution or reproduction in other forums is permitted, provided the original author(s) and the copyright owner(s) are credited and that the original publication in this journal is cited, in accordance with accepted academic practice. No use, distribution or reproduction is permitted which does not comply with these terms.

\section{YOUNG REVIEWER}

\section{UMBERTO, AGE: 10}

My name is Umberto and I like to study science, play with the Nintendo Switch with my friends. I love going to the beach to play with sand, adventures, and drawing cartoons. In the future I would like to become a physicist.

\section{AUTHORS}

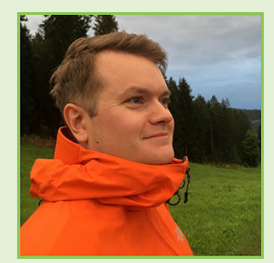

\section{DAVID REHNLUND}

I am a post-doctoral researcher at Karlsruhe Institute of Technology and Uppsala University. In the lab, I study both electrical materials and electrical bacteria. In my battery research, I study how we can improve our reversible lithium ion batteries to make them store more energy for a longer time. I also study how electroactive bacteria can communicate with metals and minerals and how this can be used to make electricity from wastewater. Outside the lab, I like to experiment with baking and enjoy fun outdoor activities, such as stand-up paddling and hiking. *david.rehnlund@akemi.uu.se 


\section{JULIA MAIBACH}

I am a researcher at Karlsruhe Institute of Technology, where I study surfaces in lithium ion batteries and teach about surface processes and how to study them. My research group mostly works on understanding the reactions that make batteries safe to use, but we also study all kinds of other surfaces, like those used for solar cells or medical devices. When I am not in the lab, I like to explore the world with my camera and try lots of tasty foods that I then try to cook at home. 